\title{
Implicancias de la biopsia selectiva de ganglio centinela en cadena mamaria interna en el cáncer de mama
} Implications in selective sentinel node biopsy in internal
mammary chain in breast cancer

Correspondencia Claudia González Espinosa claudiage@infomed.sld.cu

Recibido: 24/02/2020

Arbitrado por pares

Aprobado: 20/05/2020

Citar como: Gonzáles Espinosa C. Valdés Guerrero 0, Campos Bernardo MC, Calderón Marín C, Mestre Fernández BF. Implicancias de la biopsia selectiva de ganglio centinela en cadena mamaria interna en el cáncer de mama. Acta Med Peru. 2020;37(2):204-8. doi: $h$ ttps://doi.org/10.35663/ amp.2020.372.1031
Claudia Gonzáles Espinosa, ${ }^{1, a}$, Orlando Valdés Guerrero ${ }^{1, b}$, María C. Campos Bernardo ${ }^{1, c}$, Carlos Calderón Marín ${ }^{1, d}$, Braulio F. Mestre Fernández ${ }^{1, e}$

Instituto Nacional de Oncología y Radiobiología. La Habana, Cuba.

Especialista en Medicina Interna; ${ }^{b}$ especialista en Oncología Médica; ${ }^{c}$ especialista en Radiología; d licenciado en Física, magíster en Física Médica; e especialista en Oncología Médica

\section{RESUMEN}

El compromiso metastásico de los ganglios linfáticos es el factor pronóstico más importante en el cáncer de mama. Conocer el grado de infiltración de los mismos implica sobrevida libre de progresión, sobrevida global y riesgo de recurrencia temprana de la enfermedad, y esto a su vez ayudaría a conocer las terapias adyuvantes que recibirán las pacientes. Presentamos un caso con diagnóstico de carcinoma ductal invasor de la mama izquierda etapa IB que se le realizó biopsia selectiva de ganglio centinela mostrando la linfocentellografía, una migración única a la cadena mamaria interna sin poder acceder al mismo por su localización anatómica retrocostal, se decide rastreo y disección axilar porque así lo recoge nuestro protocolo, a partir de casos como este, nos obliga a realizar una revisión y actualización del tema, y así reajustar nuestros protocolos de actuación con la introducción de técnicas novedosas en nuestro país y los resultados de nuestras primeras experiencias acumuladas. La cadena mamaria interna puede ser el único territorio ganglionar comprometido en la biopsia selectiva de ganglio centinela y, a la vista de los resultados, podría obviarse el estudio ganglionar a nivel axilar cuando no hay sospecha clínica ni radiológica de afectación.

Palabras clave: Biopsia del ganglio linfático centinela; Metástasis linfática; Cáncer de mama (fuente: DeCS BIREME). 


\section{ABSTRACT}

Metastatic lymph node involvement is the most important prognostic factor in breast cancer. To know the degree of infiltration of the same involved progression-free survival, global survival and risk of early recurrence of the disease, and this in turn would help to know the adjuvant therapies that patients will receive. We present a case with a diagnosis of stage IB invasive ductal carcinoma of the left breast undergoing selective sentinel lymph node biopsy showing lymphocentellography, single migration to the internal mammary gland without access to it due to its retrocostal anatomical location, and axillary exploration and dissection were decided because this is what our protocol includes, based on cases like this, it forces us to carry out a review and update of the subject, and thus readjust our action protocols with the introduction of novel techniques in our country and the results of our first accumulated experiences. The internal mammary chain may be the only lymph node territory involved in selective sentinel lymph node biopsy and, in view of the results, it could obviate lymph node study at the axillary level when there is no clinical or radiological suspicion of involvement.

Keywords: Sentinel lymph node biopsy; Lymphatic metastasis; Breast cancer (source: MeSH NLM).

\section{INTRODUCCIÓN}

La infiltración de los ganglios linfáticos es el factor pronóstico más importante en el cáncer de mama ${ }^{[1]}$. Conocer el grado de invasión metastásica de los mismos determinará, no solo el pronóstico, sino también la necesidad de terapia adyuvante asociada que recibirán las pacientes.

Desde el año 1994, se introduce la técnica de biopsia selectiva de ganglio centinela (BSGC) en el cáncer de mama como técnica de primera elección para la estadificación ganglionar, reservando la linfadenectomía axilar a determinados casos con invasión de los nodos demostrada en el estudio histológico o clínicamente o radiológicamente demostradas ${ }^{[2]}$. La localización más frecuente de los ganglios centinela en el cáncer de mama es la axila en el $99 \%$ de los casos, seguida de la cadena mamaria interna (CMI) que ocupa entre el 9-18\% de los pacientes. Conocemos que la realización de la BSGC en la CMI resulta una técnica controvertida. En los casos con drenaje del trazador tanto a axila como a CMI, la mayoría de los centros se decantan por realizar únicamente el estudio a nivel axilar, con el consecuente riesgo de infraestadificación que ello conlleva ${ }^{[3,4]}$. El problema se presenta cuando el único territorio de migración es la CMI.

El objetivo principal de este estudio es mostrar un caso clínico con diagnóstico de cáncer de mama con migración única del radiotrazador a CMI y determinar la implicación clínica, pronóstica y terapéutica de la BSGC en esta paciente sin sospecha de afectación a nivel axilar.

\section{REPORTE DE CASO}

Se trata de una paciente femenina de color de piel negra de 61 años de edad, posmenopáusica con antecedentes clínicos de hipertensión arterial hace diez años, controlada con dieta y tratamiento medicamentoso instaurado con enalapril $20 \mathrm{mg}$ diarios y obesidad grado I (índice de masa corporal de $31 \mathrm{~kg} / \mathrm{m}^{2}$ ). Con historia obstétrica: tres embarazos, dos partos y un aborto provocado, ofreciendo lactancia hasta los 6 meses a ambos hijos.
Paciente que acude a consulta de clasificación del Instituto de Oncología de la Habana por presentar un nódulo duro, móvil en proyección de la mama izquierda. Al examen físico se constata mama izquierda: piel íntegra, sin cambios de coloración ni aumento de volumen a la inspección de la mama, ni retracción del pezón. A la palpación, se detecta un nódulo de aproximadamente $2 \mathrm{~cm}$ en cuadrante superior interno, móvil, indurado mal definido al tacto, no doloroso ni se evidencia secreción por el pezón. La mama contralateral y axilas presentan una exploración clínica dentro de los límites normales.

Se le indica ultrasonido (US) y mamografía (Mx) informando como hallazgos positivos: US con nódulo sólido irregular con márgenes no circunscritas de $13 \times 10 \mathrm{~mm}$ con distorsión del patrón mamario localizado en cuadrante superior interno de la mama izquierda. Adenopatías de aspecto inflamatorias en ambas axilas, lo que corresponde a un estadio BIRADS V. Asimismo en la $\mathrm{Mx}$, se evidencia un nódulo sólido irregular y de márgenes no circunscritas de $13 \times 10 \mathrm{~mm}$ con distorsión del patrón mamario y microcalcificaciones patológicas asociadas en igual localización. Se decide realizar biopsia por aguja gruesa de la lesión de la mama, dicha biopsia informa un Carcinoma ductal invasor de alto grado con una inmunohistoquímica con las siguientes características: RE: negativo, RP: negativo, HER-2: 0, Ki-67\%: 70\%. Se realiza estudios de extensión: biometría hemática, tomografía computarizada de cráneo, torácica y abdomen y gammagrafía ósea de cuerpo entero sin alteraciones de significado oncológico.

Se decide excéresis amplia del tumor más BSGC en instancias quirúrgicas. Se remitió al servicio de medicina nuclear para la localización selectiva de ganglio centinela (GC), el día previo a la cirugía. Tras la inyección periareolar de 111 MBq de 99mTcnanocoloide de albúmina en mama izquierda de manera intradérmica en cuadrante de la lesión, se obtuvieron imágenes gammagráficas dinámicas al momento de la inyección y planares a los 30 minutos en proyecciones anterior del tórax, mostrando migración única del radiofármaco a lo que impresionaba CMI (Figura 1). Ante dicha migración de drenaje linfático se decide obtener una imagen híbrida en la tomografía computarizada de emisión de fotón único y tomografía computarizada (SPECT-TC, 
por sus siglas en inglés) observándose una clara captación a la zona paraesternal izquierda, a nivel del segundo cartílago costal aproximadamente (Figura 2). Dicha imagen no se modifica significativamente en posteriores registros, considerándose migración única a CMI homolateral. Los contajes externos, colocando la sonda detectora sobre la piel del área afectada, fueron de $850 \mathrm{cps}$. (fondo 10-15 cps).

La mañana siguiente a la realización de la prueba de detección del GC se lleva a cabo la intervención quirúrgica para la excéresis amplia del tumor, se localizó e intentó biopsiar el GC marcado en estación de mamaria interna, pero se encontraba retrocostal, de difícil acceso por lo que no se pudo obtener muestra del mismo ni su excéresis. Se optó por linfadenectomía axilar (establecida en nuestra base de datos como obtención de 6 ganglios o más), en este caso se extirparon 24 ganglios en total, 23 del nivel I y II, y un ganglio del nivel III. La biopsia intraoperatoria del tumor mamario es informada como un carcinoma ductal invasor de alto grado de malignidad SBR $(3+3+3) 9$ con rasgos metaplásicos de $2 \times 1 \times 5 \times 1 \mathrm{~cm}$ con infiltrado linfocitario peritumoral severo $y$ desmoplasia, sin permeación vascular ni sanguínea. Todos los ganglios extirpados fueron negativos de malignidad. Después del estudio anatomopatológico realizado, con la clasificación AJCC-TNM (American Joint Committe on Cancer, por sus siglas en inglés) en su octava edición (TNM8), se estadía el caso como pT1c NO M0. G3 RE: negativo, RP: negativo, HER-2: 0 etapa IB.

Cabe señalar que se obtuvo la autorización y consentimiento de la paciente para la publicación de su caso, actualmente sometida a tratamiento quimioterápico adyuvante dado los factores de riesgos presentes en la paciente como son el alto grado histológico, receptores hormonales negativos, elevada proliferación tumoral Ki $67>70 \%$ e infiltrado linfocitario peritumoral severo, y radioterápico por la cirugía conservadora llevada a cabo. Al presente con evolución estable y buena respuesta hasta el momento.

\section{DISCUSIÓN}

La CMI es el segundo territorio más frecuente de afectación linfática, después de la axila ${ }^{[4]}$, e incluso puede ser el único territorio ganglionar implicado, lo que obliga a establecer el estado de invasión ganglionar por medio del estudio de esta región. La localización del tumor a nivel profundo y medial en la mama se asocia a migración del radiotrazador a la CMI y así lo vemos en esta paciente, donde la localización del GC a nivel del cuadrante superior interno presentó una migración exclusivamente en la $\mathrm{CMI}^{[5]}$. Esta técnica es factible y accesible en manos expertas, sin alargar excesivamente el tiempo quirúrgico ${ }^{[6]}$.

Estudios que evalúan las implicaciones de la biopsia selectiva de ganglio centinela en cadena mamaria interna sobre la radioterapia adyuvante en mujeres con cáncer de mama sin invasión axilar acentúan la importancia de descartar metástasis a este nivel, pero concluye que la linfadenectomía resulta también una decisión controvertida ${ }^{[7,8]}$. En una revisión sistemática de

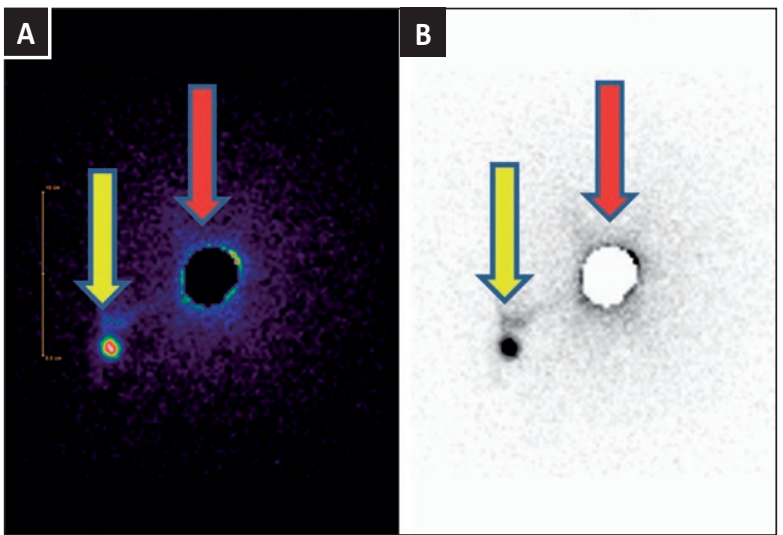

Figura 1. Imagen planar anterior gammagráfica con ${ }^{99}$ Tcnanocoloide. A en escala de colores y $B$ en escala de grises. La fecha roja muestra el sitio de inyección del radiofármaco en cuadrante superior interno de la mama izquierda y la fecha amarilla, migración a ganglio centinela a posible estación mamaria interna.

la biblioteca Cochrane acerca del tratamiento sobre la axila en pacientes con cáncer de mama en estadios precoces, cuyo objetivo es evaluar los beneficios y daños de los enfoques alternativos para la cirugía axilar en términos de supervivencia general; recaídas y eventos adversos. En aquellos casos en los que no sea posible realizar la BSGC, se acepta el muestreo ganglionar axilar ya que la efectividad en cuanto a supervivencia global es similar a la linfadenectomía, con unas tasas menores de linfedema, concluyendo y dejando de manera transparente que la linfadenectomía axilar ya no es una técnica quirúrgica aceptable en una axila clínica y radiológicamente negativa ${ }^{\left[{ }^{[9]}\right.}$. En nuestro caso se decidió el rastreo y disección axilar porque así lo recoge nuestro protocolo, siendo la paciente sobretratada a la luz de los estudios actuales, a partir de casos como el que presentamos, nos obliga a realizar una revisión y actualización de este tema, y así reajustar nuestros protocolos de actuación con la introducción de técnicas novedosas en nuestro país y los resultados de nuestras primeras experiencias acumuladas.

Autores como Heuts et al., han demostrado que existe un riesgo sustancial de hasta un $22 \%$ de posibilidad de metástasis en pacientes con migración a $\mathrm{CMI}$, con las consecuencias oportunas, sin escatimar la morbilidad asociada a la radioterapia (RT) en ocasiones innecesarias ${ }^{[10]}$. Abigail et al. plantean que la identificación de metástasis en CMI presenta un alto impacto en la planificación terapéutica, sugiriendo la excéresis siempre que sea posible en caso de drenaje a dicho nivel ${ }^{[11]}$. Puesto que la quimioterapia (QT) adyuvante ha demostrado beneficio en pacientes con cáncer de mama e indicación de recibir dicho tratamiento, la realización de la BSGC en CMI estaría aconsejada en pacientes sin criterios de QT por las características del tumor, que pueden terminar recibiéndola si se hallan metástasis ganglionares ${ }^{[12]}$.

El control locoregional del tumor reduce la mortalidad, el tratamiento de los territorios ganglionares afectos, lo que 


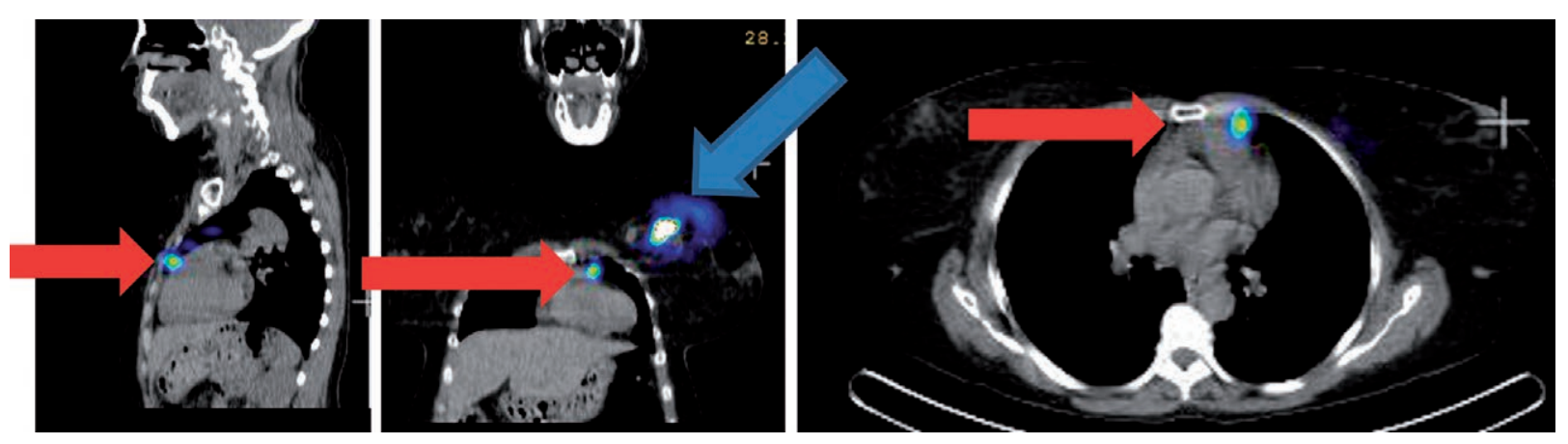

Figura 2. SPECT/CT de tórax con ${ }^{99} \mathrm{TC}-$ nanocoloide en cortes sagital, axial y coronal. La flecha roja muestra una migración a ganglio centinela a la cadena mamaria interna izquierda en las Figuras A, B y C y la flecha azul, el sitio de inyección del radiofármaco

mejoraría la supervivencia libre de enfermedad y global. Los ensayos clínicos aleatorizados MA.20 del National Cancer Institute of Canada que analiza el papel de la radioterapia ganglionar regional en el cáncer de mama tratado con cirugía conservadora ${ }^{[12]}$, AMAROS que comparó la eficacia de la linfadenectomía quirúrgica frente a la radioterapia ganglionar ( «linfadenectomía rádica») ${ }^{[13]}$, EORTC2292-10925 que compara pacientes con tumores de mama de localización central o en cuadrantes internos independientemente de la afectación axilar o en cuadrantes externos con afectación ganglionar y que recibieron de manera aleatoria radioterapia sobre la mama o la pared torácica frente al mismo tratamiento, incluyendo también la irradiación de las áreas ganglionares supraclavicular y mamaria interna ipsilateral ${ }^{[14]}$ y, más recientemente, el DBCG$\mathrm{IMN}^{[15]}$ donde sugieren que la RT aplicada sobre los ganglios del nivel III axilar, del nivel supraclavicular y de la CMI aumenta la supervivencia libre de enfermedad, la supervivencia libre de metástasis y la supervivencia global cuando estos territorios están afectados, pero si no se dispone de datos sobre invasión ganglionar a nivel de la $\mathrm{CMI}$, no se puede saber qué pacientes se estarán sobretratando.

Como mencionamos anteriormente la cadena CMl es el segundo sitio de diseminación nodal en el cáncer de mama después de la región axilar. Las pacientes con GC en CMI positivos tienen una menor sobrevida global y específica del cáncer de mama, con aumento en las tasas de metástasis a distancia, hasta hace poco tiempo la disección de la CMI o la terapia radiante no habían demostrado aumento de la sobrevida global de estas pacientes, recuperándose dicho concepto a la luz de resultados actuales antes expuestos, que han confirmado un beneficio de la RT en cuanto a sobrevida en pacientes después de 10 años de seguimiento, sin embargo, no podemos dejar de lado las complicaciones de la misma, por lo tanto, este tratamiento debe considerarse si es sustancialmente beneficioso ${ }^{[16]}$.

Con la introducción de nuevas unidades de tratamiento y nuevas modalidades de RT, como son la RT de intensidad modulada y los sistemas de control respiratorio durante la irradiación, como son el gating o la irradiación en inspiración profunda, los efectos derivados de la radiación en la región paraesternal han disminuido, pero no se deben infraestimar; por ello, es importante confirmar histológicamente la afectación de los ganglios a este nivel, siguiendo la línea europea actual indica que ante la presencia de infiltración metastásica de GC en mamaria interna sin migración del RF a región axilar o región axilar no comprometida por la enfermedad se sugiere RT adyuvante de $\mathrm{CMI}$ además del lecho quirúrgico en pacientes menores de 70 años con tumor mayor de $1,5 \mathrm{~cm}$ con factores predisponentes a cardiotoxicidad por RT, sobrepesando el beneficio de la sobrevida ${ }^{[14,17]}$.

A la vista de los estudios actuales haciendo un análisis crítico de esta paciente, nos encontramos ante un caso que fue sometido a un sobretratamiento en el manejo de la axila en una paciente con diagnóstico de cáncer de mama con edad menor a los 70 años, una lesión menor de $1,5 \mathrm{~cm}$ y $\sin$ factores que la pudieran predisponer al tratamiento rádico de la cadena mamaria interna en caso de estar comprometida, estudio que no se pudo realizar.

En conclusión, es muy importante tener en cuenta que, con la introducción de nuevas técnicas en el diagnóstico, estadiamiento y/o tratamiento del cáncer de mama se debe reconfigurar y repensar los protocolos de actuación en función de los diferentes avances, la $\mathrm{CMI}$ puede ser el único territorio ganglionar comprometido en la BSGC y, según de los resultados actuales, podría obviarse el estudio ganglionar a nivel axilar cuando no hay sospecha clínica ni radiológica de afectación. Tanto el éxito en la localización del GC como el resultado de la biopsia en CMI modifican de manera significativa la indicación de Radioterapia a este nivel, y con ello el pronóstico de sobrevida global y específica en cáncer de mama, así como el control locoregional; por lo que su búsqueda y realización no debería ser obviada.

Queda demostrado en este caso que fue sometido a sobretratamiento de la estación ganglionar axilar con sus consecuentes complicaciones a largo plazo, y maltratando la cadena mamaria interna donde no se pudo definir compromiso de la misma, dejando el pronóstico expectante inseguro para nuestra paciente.

Contribuciones de autoría: los autores declaran haber realizado contribuciones significativas a la concepción y diseño del manuscrito, la recolección de los datos, redacción y revisión 
crítica del contenido del manuscrito, la aprobación final de la versión que se publicará y asumen la responsabilidad frente a todos los aspectos del manuscrito.

Potenciales conflictos de interés.: los autores declaran no tener conflictos de intereses.

Fuente de financiamiento: autofinanciado.

\section{ORCID:}

Claudia González Espinosa: https://orcid.org/0000-0002-2106-7701 Orlando Valdés Guerrero: https://orcid.org/0000-0002-7460-8911 María C. Campos Bernardo: https://orcid.org/0000-0001-8973-8906 Carlos Calderón Marín: https://orcid.org/0000-0003-2783-1261 Braulio F. Mestre Fernández: https://orcid.org/0000-0002-6218-3653

\section{REFERENCIAS BIBLIOGRÁFICAS}

1. Veronesi U, Cascinelli N, Bufalino R, Morabito A, Greco M, Galluzzo $D$, et al. Risk of internal mammary lymph node metastases and its relevance on prognosis of breast cancer patients. Ann Surg. 1983;198(6):681-4. doi: 10.1097/00000658-198312000-00002.

2. Lyman GH, Somerfield MR, Bosserman LD, Giuliano AE. Sentinel lymph node biopsy for patients with early-stage breast cancer: American Society of Clinical Oncology Clinical Practice Guideline Update. J Clin Oncol. 2017;35(5):561-4. doi: 10.1200/ JCO.2016.71.0947.

3. Amin MB, Edge S, Greene F, Byrd DR, Brookland RK, Washington MK, et al. The Eighth Edition AJCC Cancer Staging Manual: Continuing to build a bridge from a population-based to a more "personalized" approach to cancer staging. CA Cancer J Clin. 2017;67(2):93-99. doi: 10.3322/caac. 21388 .

4. Montsech L, Torrecabota J, Bendito A, Peñafiel A. Cáncer de mama con invasión metastásica del ganglio centinela en cadena mamaria interna y cadenas axilares libres de lesión tumoral. Rev. Esp. Med. Nuclear. 2001;20(4):295-298. doi: 10.1016/S0212-6982(01)71961-1.

5. Frati A, Delpech $Y$, Bricou A, Barranger E. Sentinel lymph node detection in patients with early-stage breast cancer: which site of injection in 2011? Gynecol Obstet Fertil. 2011;39(11):620-3. doi: 10.1016/j.gyobfe.2011.07.019.

6. Ferreres García K, Siegrist Ridruejo J, Rincón Olbés P, Luque Molina MS, Almoguera Arias MI. Therapeutic attitude towards internal mammary chain drainage in patients with breast cancer. Breast. 2016;30:1-4. doi: 10.1016/j.breast.2016.08.001.
7. Ferreres García K, Luque Molina MS, Rincón Olbés $P$, Mendizábal EM, Hernández Sánchez L, Lizarraga Bonelli S. Implicaciones de la biopsia selectiva de ganglio centinela en cadena mamaria interna sobre la radioterapia adyuvante en mujeres con cáncer de mama sin invasión axilar. Rev Senol Patol Mamar. 2018;31(2):54-58. doi: 10.1016/j.senol.2018.03.006.

8. Van Esser S, Madsen EVE, van Dalen T, Koelemij R, van Rossum PSN, Borel Rinkes IHM, et al. Axillary staging in breast cancer patients with exclusive lymphoscintigraphic drainage to the internal mammary chain. World J Surg. 2011;35:159-64.

9. Bromham N, Schmidt-Hansen M, Astin M, Hasler E, Reed MW. Axillary treatment for operable primary breast cancer. Cochrane Database Syst Rev. 2017. doi: 10.1002/14651858.CD004561. pub3.

10. Heuts EM, van der Ent FW, von Meyenfeldt MF, Voogd AC. Internal mammary lymph drainage and sentinel node biopsy in breast cancer - A study on 1008 patients. Eur J Surg Oncol. 2009;35(3):252-257. doi:10.1016/j.ejso.2008.06.1493.

11. Caudle AS, Yi M, Hoffman KE, Mittendorf EA, Babiera GV, Hwang RF, et al. Impact of IM SLN on Breast Cancer Therapy. Ann Surg Oncol. 2014;21(1):60-65. doi: 10.1245/s10434-013-3276-z.

12. Whelan TJ, Olivotto IA, Parulekar WR, Ackerman I, Chua BH, Nabid $A$, et al. Regional nodal irradiation in early-stage breast cancer. $\mathrm{N}$ Engl J Med. 2015;373:307-16. doi: 10.1056/NEJMoa1415340.

13. Donker $\mathrm{M}$, van Tienhoven $\mathrm{G}$ et al. Radiotherapy or surgery of the axilla after a positive sentinel node in breast cancer (EORTC 10981-22023 AMAROS): A randomised, multicentre, open-label, phase 3. Lancet Oncol. 2014;15(12):1303-10. doi: 10.1016/\$14702045(14)70460-7.

14. Poortmans PM, Collette S, Kirkove C, van Limbergen E, Budach V, Struikmans H, et al. EORTC Radiation Oncology and Breast Cancer Groups. Internal mammary and medial supraclavicular irradiation in breast cancer. N Engl J Med. 2015;373:317-27. doi: 10.1056/ NEJMoa1415369.

15. Thorsen LBJ, Offersen BV, Dan $\varnothing \mathrm{H}$, Berg $M$, Jensen I, Pedersen AN, et al. DBCG-IMN: A population-based cohort study on the effect of internal mammary node irradiation in early nodepositive breast cancer. J Clin Oncol. 2016;34(4):314-20. doi: 10.1200/ JCO.2015.63.6456.

16. Van Loevezijn AA, Bartels SAL, van Duijnhoven FH, Heemsbergen WD, Bosma SCJ, Elkhuizen PHM et al. Internal Mammary Chain Sentinel Nodes in Early-Stage Breast Cancer Patient: Toward Selective Removal. Ann Surg Oncol. 2019;26(4):945-953. doi: 10.1245/s10434-018-7058-5.

17. Morigi C. Highlights from the 15th St Gallen International Breast Cancer Conference 2017 Mar 15-18 March, 2017, Vienna: tailored treatments for patients with early breast cancer. Ecancermedicalscience. 2017;11:732. doi: 10.3332/ ecancer.2017.732. 